\title{
COMBINING DECODED-AND-FORWARDED SIGNALS IN GAUSSIAN COOPERATIVE CHANNELS
}

\author{
B. Djeumou, S. Lasaulce, A. G. Klein \\ LSS CNRS, 3 rue Joliot-Curie, 91190 Gif-sur-Yvette, France, \{djeumou,lasaulce,klein\} @1ss.supelec.fr
}

\begin{abstract}
The goal of this paper is to investigate different ways of combining signals that have been decoded-and-forwarded by a bunch of relays. We more deeply look at the case where the relays are in bad reception conditions and the cooperation powers are sufficiently high. In this situation using a conventional MRC severely degrades the receiver performance especially when the number of relays increases. On the other hand the MMSE- and ML-based combiners can almost always extract from their partners some performance improvements.
\end{abstract}

Keywords -Relay channel, Cooperation, Decode-and-forward, MRC, MMSE, ML

\section{INTRODUCTION}

The relay channel was introduced by [1]. In its most basic form it consists of one transmitter, one relay node and one receiver. The main issue is to know what the relay does to maximize the receiver performance (in terms of data rate and/or error probability). In this respect two important concepts have been introduced in the seminal information-theoretic work [2] by Cover and El Gamal : Decode-and-foward (DF) and estimate-and-forward (EF). In [3] the authors studied linear relaying schemes, also from the channel capacity point of view. The corresponding scheme is commonly referred to as amplify-and-forward (AF). There have been numerous works on the comparison between these three relaying strategies (AF, $\mathrm{DF}, \mathrm{EF})$ both in terms of achievable rates and bit error rates.

In this paper we assume a given choice for the relaying scheme : only decode-and-forward is considered. The channel under investigation is quite similar to that studied in [4], which is called a Gaussian relay network. In this paper the destination receives one direct signal from the source and several decodedand-forwarded versions of the transmitted signal. Also these signals are assumed to be orthogonal. In this context we want to know how to combine the received signals and evaluate the maximum performance improvement that be extracted from relaying in any situation, especially when the relays have receive signal-to-noise ratios (SNR) comparable to (or less than) the signal-to-noise ratio at the receiver. While the informationtheoretic version of DF [2] suggests that nothing can be gained from cooperation when the link between the transmitter and the relay is "worse" than that between the transmitter and the receiver, we will see that bit error rate (BER) analyses can lead to a different conclusion especially when the number of relays increases.

Usually the relay is assumed to be "between" the transmitter and the receiver so that it has a much better receive SNR. In order to make certain cooperation coding schemes work this assumption is even necessary [5]. Under this assumption, com- bining the direct and DF-based cooperation signals by using the well-known maximum ratio combining (MRC) is a good choice. It is quasi-optimal in terms of equivalent SNR and therefore in terms of BER. But, as our analysis will show, it is much less efficient when the relay has a "low" SNR. In order to improve the combining strategy we will assume that the receiver is informed with the decoding reliability at the relay. To the authors' knowledge the closest work (to the present paper) using this approach is [6] where the authors designed energyefficient transmission protocols for the fading relay channel. Our goal is then to see to what performance improvements this a priori knowledge can lead to. For this purpose we will first present our signal model (section II.) and provide different combining schemes that exploit this a priori knowledge (section III.). In section IV. we will assess the performance of the proposed schemes compared to the conventional MRC solution. Conclusions and suggested extensions are given in section V..

\section{SIGNAL MODEL}

The channel studied in this paper is illustrated in Fig. 1. At the source the information message (information bits) $W$ is encoded and modulated into the transmitted signal $X \in \mathcal{X}$ with $E\left[\left|X^{2}\right|\right] \leq P_{0}$. Most of the results in this paper hold for any type of constellations for $X$ but for simplicity we will sometimes assume $\mathcal{X}$ to represent a BPSK modulation i.e. $\mathcal{X}=\left\{-\sqrt{P_{0}},+\sqrt{P_{0}}\right\}$. All the links defining the overall channel are assumed to be AWGN channels : For all $i^{\prime} \in\{1, \ldots, N\}$ and $i \in\{0, \ldots, N\}, h_{i}^{\prime}$ and $h_{i}$ are constant complex scalars known to the relays and receiver respectively (for insuring coherent decoding); $\forall i^{\prime} \in\{1, \ldots, N\}, Z_{i}^{\prime} \sim \mathcal{N}\left(0, N_{i}^{\prime}\right)$, $\forall i \in\{0, \ldots, N\}, Z_{i} \sim \mathcal{N}\left(0, N_{i}\right)$. The signals forwarded by the relays are denoted by $X_{i}$ for all $i \in\{1, \ldots, N\}$ and are subject to power constraints : $\forall i \in\{1, \ldots, N\}, E\left[\left|X_{i}\right|^{2}\right] \leq P_{i}$. The received signals merely write as :

$$
\forall i \in\{0, \ldots, N\}, \quad Y_{i}=h_{i} X_{i}+Z_{i}
$$

These signals are assumed to be othogonal, which can be implemented by using time or frequency division. As the ressource allocation issue is not addressed in this paper we did not introduce in our notations any time sharing or bandwidth fraction parameter. In the paper we will also make use of the following quantities $: \forall i^{\prime} \in\{1, \ldots, N\}, \rho_{i}^{\prime}=P_{0} / N_{i}^{\prime}$ and $\forall i \in\{0, \ldots, N\}, \rho_{i}=P_{i} / N_{i}$. 


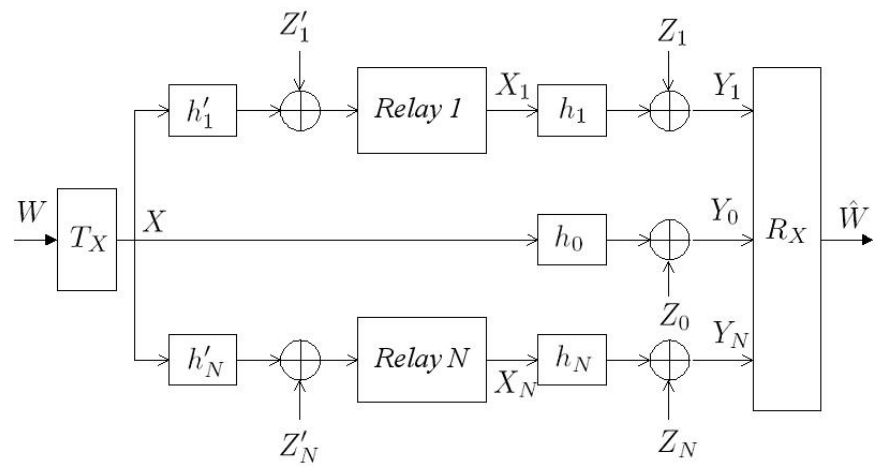

FIG. 1 - Channel under investigation

\section{SIGNAL COMBINING SCHEMES}

The main point is therefore how to combine the received signals in order to minimize the bit error rate for the receiver under consideration. To this end we will assume perfect knowledge of the different useful channels and SNRs at each receiver and discuss this assumption further.

Optimality of combining will be defined with respect to three criteria : the SNR at the output of a linear combiner, the MMSE at the output of a linear combiner and the ML criterion as a non-linear detector. One of the reasons why we considered both solutions is that we want to take advantage of cooperation as much as possible namely even if the relay-receiver is in "bad" reception conditions. For instance, it is generally thought that using DF when SNR at the relay is close or less than that of the destination is useless. We will see it is not always the case.

\section{A. Maximum ratio combiner}

As each relay " $i$ " is assumed to decode-and-forward what it receives the cooperation signal $X_{i}$ corresponds to demodulating, decoding, re-encoding and re-modulating the information bits sent by the transmitter. Usually the receive relay SNR $\rho_{i}^{\prime}\left|h_{i}^{\prime}\right|^{2}$ is assumed to be high enough so that the decoding errors can be neglected (see e.g. [5]), which amounts to assuming $X_{i} \simeq \alpha_{i} X$ in the signal model, with $\alpha_{i}=\sqrt{\frac{P_{i}}{P_{0}}}$. By using the corresponding approximated signal model the weight vector maximizing the SNR at the output of the MRC is known to be :

$$
\underline{w}_{m r c}^{(c o n v)}=\beta \mathbf{R}_{z z}^{-1} \underline{h}^{*}
$$

where $\beta$ is an arbitrary (positive) scaling factor, $\mathbf{R}_{z z}=E\left[\underline{Z Z}^{H}\right], \quad \underline{Z}=\left[Z_{0}, Z_{1}, \ldots, Z_{N}\right]^{T}$ and $\underline{h}=\left[h_{0}, \alpha_{1} h_{1}, \ldots, \alpha_{N} h_{N}\right]^{T}$.

Now we want to evaluate the impact of this approximation i.e. the performance loss induced by using the conventional MRC weights in presence of decoding errors at the relay. For making interpretations easier we study the case where only one relay is available $(N=1)$ and a BPSK modulation is used at the transmitter and the relay. Assuming the noises to be decorrelated the optimal weights can be expressed as :

$$
\left\{\begin{array}{l}
w_{0}^{(\text {conv })}=\frac{h_{0}^{*}}{N_{0}} \\
w_{1}^{(\text {conv })}=\frac{\alpha_{1} h_{1}^{*}}{N_{1}}
\end{array}\right.
$$

with $\alpha_{1} \triangleq \sqrt{\frac{P_{1}}{P_{0}}}$. Although the conventional MRC weights are calculated from the approximated signal model we have to use the exact signal model in order to evaluate the equivalent SNR at the MRC output. We have $Y_{0}=h_{0} X+Z_{0}, Y_{1}=h_{1} X_{1}+Z_{1}$ with $X_{1}=\alpha_{1}(X+\delta X)$. For a BPSK modulation $\delta X$ is a white random variable such that $\operatorname{Pr}[\delta X=0 \mid X]=1-p_{1}$, $\operatorname{Pr}\left[\delta X=+2 \sqrt{P_{0}} \mid X=-\sqrt{P_{0}}\right]=\operatorname{Pr}\left[\delta X=-2 \sqrt{P_{0}} \mid X=\right.$ $\left.+\sqrt{P_{0}}\right]=p_{1} / 2$ where $p_{1}$ is the residual bit error rate (after re-encoding) at the relay. Putting the demodulation noise due to the relay in the noise part the equivalent noise writes $Z_{1}^{\prime}=$ $Z_{1}+\alpha_{1} h_{1} \delta X$. The equivalent SNR at the output of the MRC using conventional weigths is then given by :

$$
S N R_{e q}^{(c o n v)}=\rho_{0}\left|h_{0}\right|^{2} \times \frac{\left(1+\frac{N_{0}\left|h_{1}\right|^{2}}{N_{1}\left|h_{0}\right|^{2}} \alpha_{1}^{2}\right)^{2}}{1+\frac{N_{0}\left|h_{1}\right|^{2}}{N_{1}\left|h_{0}\right|^{2}} \alpha_{1}^{2}+\frac{4 P_{0} N_{0}\left|h_{1}\right|^{4} p_{1}}{N_{1}^{2}\left|h_{0}\right|^{2}} \alpha_{1}^{4}} .
$$

It turns out that this SNR does not always increase with the cooperation power $P_{1}$ for a fixed transmit power $P_{0}$ or with $P_{0}$ for a fixed $P_{1}$. This phenomenon arises when $p_{1} \geq \frac{1}{8 \rho_{0}\left|h_{0}\right|^{2}}$. This effect becomes more and more acute as the cooperation power increases :

$$
\lim _{P_{1} \rightarrow \infty} S N R_{e q}^{(\text {conv })}=\frac{1}{4 p_{1}},
$$

which means that the equivalent SNR becomes bounded in the cooperation and transmit powers. This is due do the fact that the effect of decoding errors at the relay are ignored and then nothing is done at the receiver to compensate for possible error amplification by the cooperation channel.

As a first step to improve the MRC performance we assume from now on that the receiver knows $p_{1}$. The purpose for having an enhanced combiner is to improve the receiver performance in "bad" receiver conditions for the relay. Otherwise there can be a non-negligible performance loss by not using the unreliably decoded signals especially when the number of relaying nodes increases. By calculating the optimum weights from the original signal model we get :

$$
\left\{\begin{array}{l}
w_{0}^{(\text {new })}=\frac{h_{0}^{*}}{N_{0}} \\
w_{1}^{(\text {new })}=\frac{\alpha_{1} h_{1}^{*}}{N_{1}+4 P_{1}\left|h_{1}\right|^{2} p_{1}}
\end{array}\right.
$$

and the corresponding equivalent SNR is

$$
S N R_{e q}^{(\text {new })}=\rho_{0}\left|h_{0}\right|^{2}+\frac{\rho_{1}\left|h_{1}\right|^{2}}{1+4 p_{1} \rho_{1}\left|h_{1}\right|^{2}} .
$$

Now we have

$$
\lim _{P_{1} \rightarrow \infty} S N R_{e q}^{(n e w)}=\rho_{0}\left|h_{0}\right|^{2}+\frac{1}{4 p_{1}} .
$$


This time, in the high cooperation regime, the equivalent SNR is not bounded in the transmit power. But if we would look at the BER performance, there would still be a problem : the raw BER is not a monotonously decreasing function of the equivalent SNR. Because of the decoding errors the relay is in fact equivalent to an additional channel. Indeed the received signal can always be rewritten as $Y_{0}=h_{0} X+Z_{1}, Y_{1}=$ $h_{1} \epsilon_{1} \alpha_{1} X+Z_{1}$ where $\epsilon_{1} \in \mathbb{C}$. The equivalent channel $\epsilon_{1}$ varies from symbol to symbol. As the instantaneous value of $\epsilon_{1}$ is unknown from the receiver, the equivalent channel $\epsilon_{1}$ cannot be "equalized". As as consequence increasing the SNR at the output of the conventional MRC does not imply decreasing the corresponding raw bit error rate. Now that we have clearly identified that we have to deal with an "equalization" problem it is clear that maximizing the SNR at the MRC ouput is not always optimal in terms of BER and therefore other performance criteria have to be used. This is the purpose of the following sections.

\section{B. MMSE combiner}

Without addtional a priori knowledge the most efficient combining scheme is the maximum likelihood (ML) detector which maximizes $p\left(y_{0}, \ldots, y_{N} \mid x\right)$. Before presenting the corresponding detector we will present the linear MMSE (minimim mean square error) detector which minimizes $E[\mid X-$ $\left.\left.\underline{w}^{T} \underline{Y}\right|^{2}\right], \underline{Y} \triangleq\left[Y_{0}, \ldots, Y_{N}\right]^{T}$ because the corresponding solution is easy to find in any context (arbitrary modulation, arbitrary decoder structure, etc.) and also to compare its performance to the ML detector. Also in certain contexts evaluating the minimum mean square error can be used, for example, to select the "best" relaying protocol. The optimum MMSE weights can be shown to be :

$$
\underline{w}_{m m s e}=\mathbf{R}_{y y}^{-1} \underline{\mu}
$$

with $\forall(i, j) \in\{0, \ldots, N\}^{2}$,

$$
R_{y y}(i, j)=\mid \begin{array}{ll}
\alpha_{i}^{2}\left|h_{i}\right|^{2} E\left[\left|\epsilon_{i}\right|^{2}|X|^{2}\right]+N_{i} & \text { if } \mathrm{i}=\mathrm{j} \\
\alpha_{i} \alpha_{j} h_{i}^{*} h_{j} E\left[\epsilon_{i}^{*} \epsilon_{j}|X|^{2}\right] & \text { if } \mathrm{i} \neq \mathrm{j}
\end{array}
$$

and $\forall i \in\{0, \ldots, N\}, \mu_{i}=\alpha_{i} h_{i}^{*} E\left[\epsilon_{i}^{*}|X|^{2}\right]$. Note that we used the notation $\alpha_{i}=P_{i} / P_{0}$. The above different terms can be easily expressed in general. For example, when $N=1$ and a M-PSK is used, we have : $E\left[\left|\epsilon_{i}\right|^{2}|X|^{2}\right]=E\left[\left|\epsilon_{i}\right|^{2} P_{0}\right]=P_{0}$, $E\left[\epsilon_{i}^{*} \epsilon_{j}|X|^{2}\right]=E\left[\epsilon_{i}^{*} \epsilon_{j} P_{0}\right]=E\left[\epsilon_{i}^{*}\right] E\left[\epsilon_{j}\right] P_{0}$ (the decoding errors at the different relays are assumed to be independent). So the key quantity is the expection $E\left[\epsilon_{i}\right]$. For the reader information the general expression of this quantity is provided in the Appendix for a general M-PSK modulation. Here we consider for simplicity the BPSK case $(M=2)$. Assuming $N=1, M=2$ the MMSE weights (eq. (9)) become :

$$
w_{0}=\frac{\left[1+4 p_{1}\left(1-p_{1}\right) \rho_{1}\left|h_{1}\right|^{2}\right] \rho_{0} h_{0}^{*}}{1+\left|h_{1}\right|^{2} \rho_{1}+\left|h_{0}\right|^{2} \rho_{0}+4\left|h_{1}\right|^{2}\left|h_{0}\right|^{2} \rho_{1} \rho_{0} p_{1}\left(1-p_{1}\right)}
$$

$$
w_{1}=\frac{\left(1-2 p_{1}\right) \rho_{1} \sqrt{\frac{P_{0}}{N_{1}}} h_{1}^{*}}{1+\left|h_{1}\right|^{2} \rho_{1}+\left|h_{0}\right|^{2} \rho_{0}+4\left|h_{1}\right|^{2}\left|h_{0}\right|^{2} \rho_{1} \rho_{0} p_{1}\left(1-p_{1}\right)} \text {. }
$$

These weights have at least two desirable properties :

- When the information from the relay-receiver is no better than a coin toss $\left(p_{1}=1 / 2\right)$, the relayed decisions are useless : $w_{1}=0$.

- When the relay always makes errors $\left(p_{1}=1\right)$ we would expect the optimal weights to be the same as the classical MMSE but with $w_{1}$ negated : $w_{1}=-\frac{\rho_{1} h_{1}^{*}}{1+\left|h_{1}\right|^{2} \rho_{1}+\left|h_{0}\right|^{2} \rho_{0}}$. We will see in the simulation part that using the MMSE combiner instead of the MRC brings some performance improvements. But, once again, it could be shown that the raw BER is not always a monotonously decreasing function of the minimum mean square error. In fact there exists a weight pair providing a better BER performance but it cannot be explicited in general. In the special case under consideration $(N=1, M=$ 2 ) it can be found numerically by minimizing the raw BER expression. Note that the corresponding combining scheme has been used by [7] for the cooperative multiple access channel under the name $\lambda-\mathrm{MRC}$. By minimimizing the raw BER with respect to $\left(w_{1}, w_{2}\right)$ one obtains the optimum linear combiner. The obtained performance is generally close to that achieved by the ML (we will not provide the corresponding simulations here but this assertion has been verified). The main issue is that one is not always able to explicit the raw BER as a function of $\left(w_{1}, w_{2}\right)$ whereas the likelikood calculation is more systematic. Additionally, when some a priori knowledge is available (say when a soft input soft output stage follows the combiner), the ML metric can be used to calculate an a posteriori probability (APP).

\section{ML combiner}

As in [6], we will always assume in this section a BPSK modulation and $h_{i} \in \mathbb{R}$ in order to provide a simple expression for the log-likelihood ratio (LLR). But the number of relays can be arbitrarily chosen. The LLR at the receiver is defined by :

$$
\lambda\left(y_{0}, \ldots, y_{N}\right)=\ln \left\{\frac{p\left(y_{0}, \ldots, y_{N} \mid X=+\sqrt{P_{0}}\right)}{p\left(y_{0}, \ldots, y_{N} \mid X=-\sqrt{P_{0}}\right)}\right\} .
$$

Under the assumption that the decoding noises at the relays are independent the LLR can be shown to be :

$$
\frac{\lambda\left(y_{0}, \ldots, y_{N}\right)}{2}=\frac{y_{0} \sqrt{\left|h_{0}\right|^{2} P_{0}}}{N_{0}}+\sum_{i=1}^{N} f_{p_{i}}\left(\frac{y_{i} \sqrt{\left|h_{i}\right|^{2} P_{i}}}{N_{i}}\right)
$$

where

$$
f_{p_{i}}(t)=\frac{1}{2} \ln \left[\frac{\left(1-p_{i}\right) e^{2 t}+p_{i}}{\left(1-p_{i}\right)+p_{i} e^{2 t}}\right] .
$$

We see that the function $f_{p_{i}}(t)$ serves as a limiter which minimizes the contribution from the relays when it is unreliable. At least four special case are worth being considered to interpret the behavior of the ML detector : 
- When $p_{i}=0, f_{p_{i}}(t)=t$ : the ML coincides with the conventional MRC.

- When $p_{i}=1 / 2, f_{p_{i}}(t)=0$ : when the information from the relay " $i$ " is no better than a coin toss, the corresponding cooperation signal is ignored.

- When $p_{i}=1, f_{p_{i}}(t)=-t$ : when the relay "i" always makes errors the ML reverses the signum of the cooperative signal.

- When the contribution of the relay is very large, for example when $P_{i}$ is very large, $f_{p_{i}}\left(\frac{y_{i} \sqrt{\left|h_{i}\right|^{2} P_{i}}}{N_{i}}\right) \simeq$ $\frac{1}{2} \ln \left(\frac{1-p_{i}}{p_{i}}\right) \operatorname{sign}\left(y_{i}\right)$ : for large cooperation powers it becomes optimal to make a decision on $y_{i}$ before combining.

\section{Simulation RESUlts}

First we assume that $h_{0}=\ldots=h_{N}=1$. All the simulations have been performed for the three cases : $N=0, N=1$ and $N=4$. We only considered the uncoded case but the performance analysis can be extended to the coded case, at least for hard input decoders. For the first three figures (Fig. 2, Fig. 3, Fig. 4) the transmit power is fixed. They represent the raw BER at the receiver as a function of the cooperation powers. The cooperation powers are assumed to be the same for all the relays : $\rho_{1}=\ldots=\rho_{N}=\rho_{\text {coop }}$ with $\left[\rho_{\text {coop }}\right]_{d B} \in[-20,40]$. Figure 2 corresponds to the case where the relays are "good": $\left[\rho_{i}^{\prime}\right]_{d B}=\left[\rho_{0}\right]_{d B}+8$. Figure 3 corresponds to the case of "medium" quality relays : $\left[\rho_{i}^{\prime}\right]_{d B}=\left[\rho_{0}\right]_{d B}$. Figure 4 corresponds to the case of "bad" relays : $\left[\rho_{i}^{\prime}\right]_{d B}=\left[\rho_{0}\right]_{d B}-8$.

As expected there is almost no difference between the conventional MRC, MMSE combiner and the ML combiner performance when the relays have better reception conditions than the receiver. The BER difference is almost zero for $N=1$ but becomes more significant as the number of relays grows. For "medium" quality relays however the MRC does not provide any performance improvement at the receiver in the high cooperation regime $\left(\left[\rho_{c o o p}\right]_{d B}>10\right.$ in the figure 3$)$ whereas the ML combiner allows for a significant decrease of the BER $\left(5.10^{-3}\right.$ instead of $2.10^{-2}$ for $N=1$ and $10^{-4}$ instead of $2 \cdot 10^{-3}$ for $N=4$ ). For the case of "bad" relays in the high cooperation regime the receiver performance using a $\mathrm{MRC}$ is dominated by the performance of the relays i.e. there is a significant performance degradation with respect to case where the receiver is alone (no relays). But the ML combiner provides a certain improvement over the no cooperation case $\left(B E R \simeq 10^{-4}\right.$ instead of $\left.B E R \simeq 9.10^{-4}\right)$.

In order to have a more complete picture of the comparison between the different combining scheme we now fix the cooperation powers $P_{1}=\ldots=P_{N}=P_{\text {coop }}$ with $\left[\frac{P_{\text {coop }}}{P_{0}}\right]_{d B}=30$ and look at the BER variations versus $P_{i} / N_{0}$ by varying $N_{0}$. Note that we consider the high cooperation regime : Such a regime can appear when the link budgets corresponding to the cooperation channels are much better than that corresponding to the downlink channels, which is in fact a very common scenario in a cellular networks (e.g. when several users are in the same room or building). Figures 5, 6, 7 represent the BER as a function of $P / N_{0}$ for the same scenarios as considered before ("good" relays, "medium" relays, "bad" relays). The most informative situations are that of figures 6,7 . For the case of "medium" quality relays : With respect to the conventional MRC the ML combiner provides a typical gain of $2 \mathrm{~dB}$ on the SNR. For the "bad" relays the MRC severely degrades the BER performance with respect to non-cooperating case (no relay) while the ML still offers a positive gain on the SNR. To sum up we see that by using the ML the receiver performance never degrades whatever the reception conditions at the relays. As for the MMSE performance it is always better than that of the conventional MRC but does not perform as well as the ML especially when the number of relays is low. Otherwise the MMSE is also a good candidate for combining unreliable decoded-and-forwarded signals.

\section{CONClusions}

We have seen that combining decoded-and-forwarded signals is beneficial even if the relays have reception conditions worse than than that of the receiver. The corresponding improvement cannot be extracted from the relays by a conventional MRC at the receiver. More efficient combining schemes have to be used such as the MMSE combiner and especially the ML combiner. Using the ML allows us to obtain a better BER performance whatever the receive conditions at the relays. We also mentioned that the present performance analysis can be readily extended to the coded case at least for hard input channel decoders. For soft input decoders (e.g. turbo-decoders) the ML combiner has to extended to an APP-based combiner. As for the MMSE it is generally less efficient but has the advantage that it is simple to find for any kind of decoders (hard or doft inputs) and any modulation scheme.

\section{ACKNOWLEDGMENTS}

The authors would like to thank Julien Dumont for providing some simulation tools used in this work.

\section{REFERENCES}

[1] E. C. Van der Meulen, "Three-terminal communication channels", Adv Appl. Proba., vol.3, pp. 120-154, 1971.

[2] T. M. Cover and A. A. El Gamal, "Capacity theorems for the relay channel”, IEEE Trans. Inform. Theory, IT-25(5) : 572-584, 1979.

[3] S. Zahedi, M. Mosheni and A. A. El Gamal, "On the capacity of AWGN relay channels with linear relaying functions", Proc. of ISIT, Chicago, June 2004.

[4] M. Gastpar and M. Vetterli, "On the capacity of large Gaussian relay networks", IEEE Trans. Inform. Theory, IT-51(3) : 765-779, 2005.

[5] B. Zhao and M. C. Valenti, "Distributed turbo coded diversity for the relay channel", IEE Electronics Letters, vol.39, pp. 786-787, May 2003.

[6] N. Laneman and G. Wornell, "Energy-efficient antenna sharing and relaying for wireless networks", Proc. of IEEE Wireless Communications and Networking Conference (WCNC), Chicago, 2000. 
[7] A. Sendonaris, E. Erkip and B. Aazhang, "User cooperation diversityPart II : Implementation aspects and performance analysis", IEEE Trans. on Comm., COM-51(11) : 1939-1948, 2003.

\section{APPENDIX}

Here we show how to calculate the expectation of the multiplicative term modelling the relay decision in the M-PSK modulation case. For sake of clarity we will omit the relay index "i" : $\epsilon_{i} \rightarrow \epsilon, p_{i} \rightarrow p$. We need to find for each symbol $k \in\left\{1, \ldots, e^{j(M-1) \frac{2 \pi}{M}}\right\}$ a serie $c_{k}(0), \ldots, c_{k}(M-1)$ such that :

$$
E[\epsilon]=\frac{1}{M} \sum_{k=0}^{M-1} \sum_{m=0}^{M-1} e^{j m \frac{2 \pi}{M}} p^{c_{k}(m)}(1-p)^{\log _{2}(M)-c_{k}(m)} .
$$

The series depend on the mapping of the information (possibly encoded) bits into the transmitted symbols. In fact the series are identical for $M=2$ and $M=4$ but differ for higher modulation order. The following table provides $E[\epsilon]$ for different values of $M$ when a Gray mapping is used. The expressions are exact for $M \in\{2,4\}$ and are approximated at the first order (in $p$ ) for $M \in\{8,16,32,64\}$.

\begin{tabular}{|c|c|}
\hline $\mathbf{M}$ & $\mathbf{E}[\epsilon]$ \\
\hline 2 & $1-2 p$ \\
\hline 4 & $1-2 p$ \\
\hline 8 & $1-\left(3-\frac{\sqrt{2}}{2}\right) p \simeq 1-2.29 p$ \\
\hline 16 & $1-\left[4-\frac{3}{2} \cos \left(\frac{\pi}{8}\right)-\frac{1}{2} \cos \left(\frac{3 \pi}{8}\right)\right] p$ \\
& $\simeq 1-2.42 p$ \\
\hline 32 & $1-\left[5-\frac{7}{4} \cos \left(\frac{\pi}{16}\right)-\frac{3}{4} \cos \left(\frac{3 \pi}{16}\right)\right.$ \\
& $\left.-\frac{1}{4} \cos \left(\frac{5 \pi}{16}\right)-\frac{1}{4} \cos \left(\frac{7 \pi}{16}\right)\right] p$ \\
& $\simeq 1-2.47 p$ \\
\hline 64 & $1-\left[6-\frac{15}{8} \cos \left(\frac{\pi}{32}\right)-\frac{7}{8} \cos \left(\frac{3 \pi}{32}\right)\right.$ \\
& $-\frac{3}{8} \cos \left(\frac{5 \pi}{32}\right)-\frac{3}{8} \cos \left(\frac{7 \pi}{32}\right)-\frac{1}{8} \cos \left(\frac{9 \pi}{32}\right)$ \\
& $\left.-\frac{1}{8} \cos \left(\frac{11 \pi}{32}\right)-\frac{1}{8} \cos \left(\frac{13 \pi}{32}\right)-\frac{1}{8} \cos \left(\frac{15 \pi}{32}\right)\right]$ \\
& $\simeq 1-2.49 p$ \\
\hline
\end{tabular}

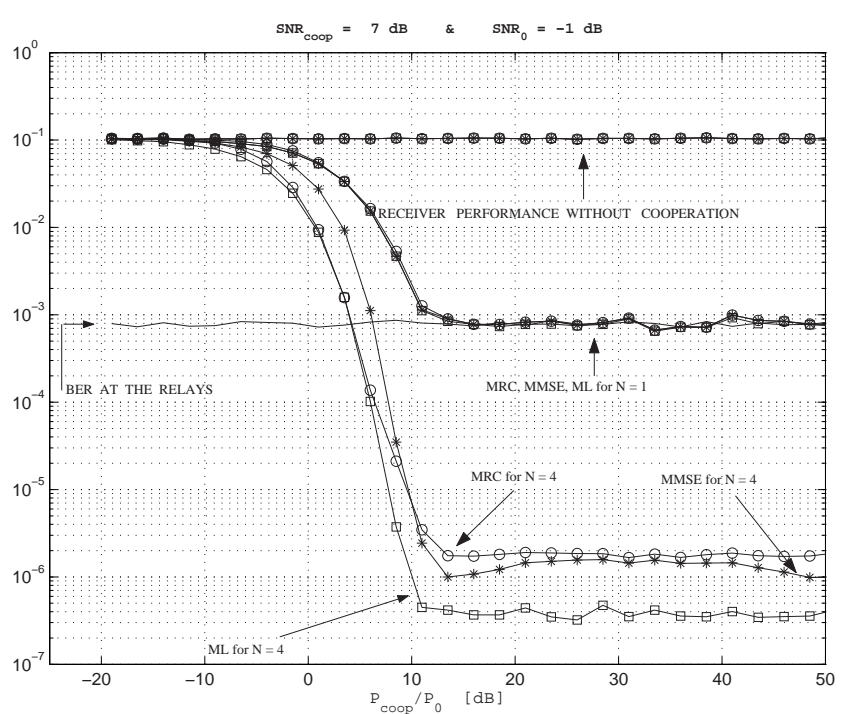

FIG. 2 - BER vs cooperation power in the case of "good" relays

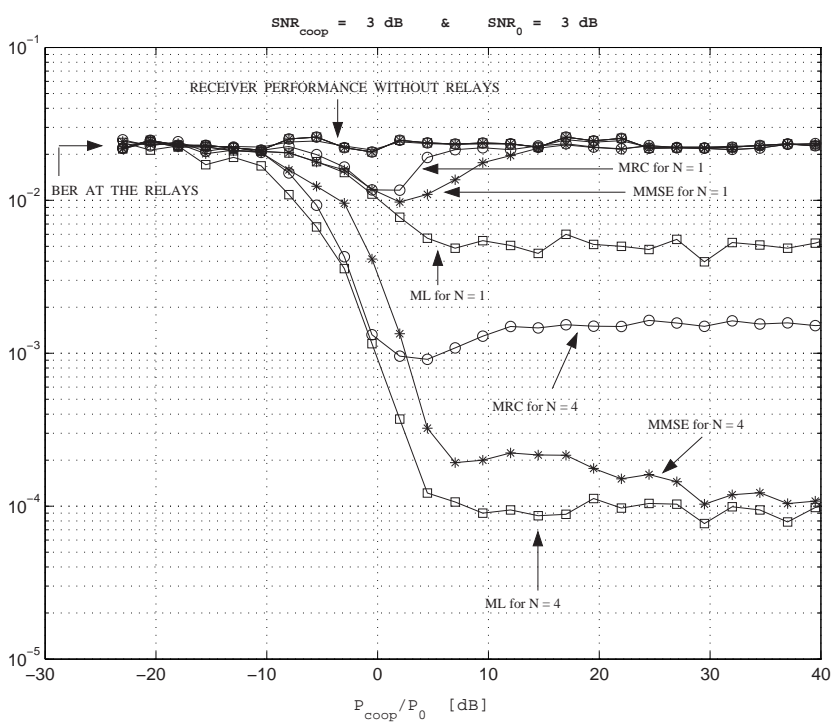

FIG. 3 - BER vs cooperation power in the case of "medium" relays

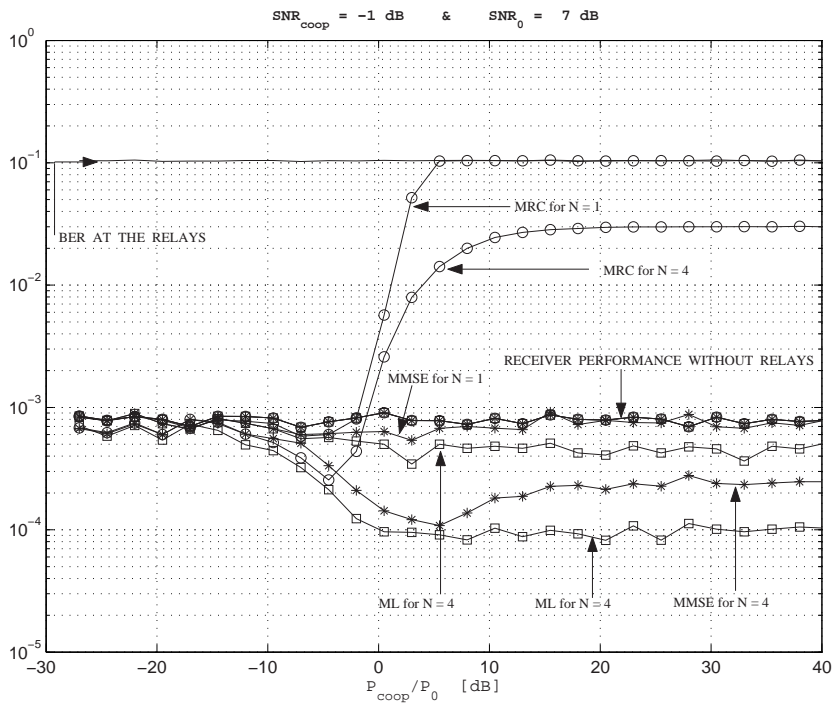

FIG. 4 - BER vs cooperation power in the case of "bad" relays 


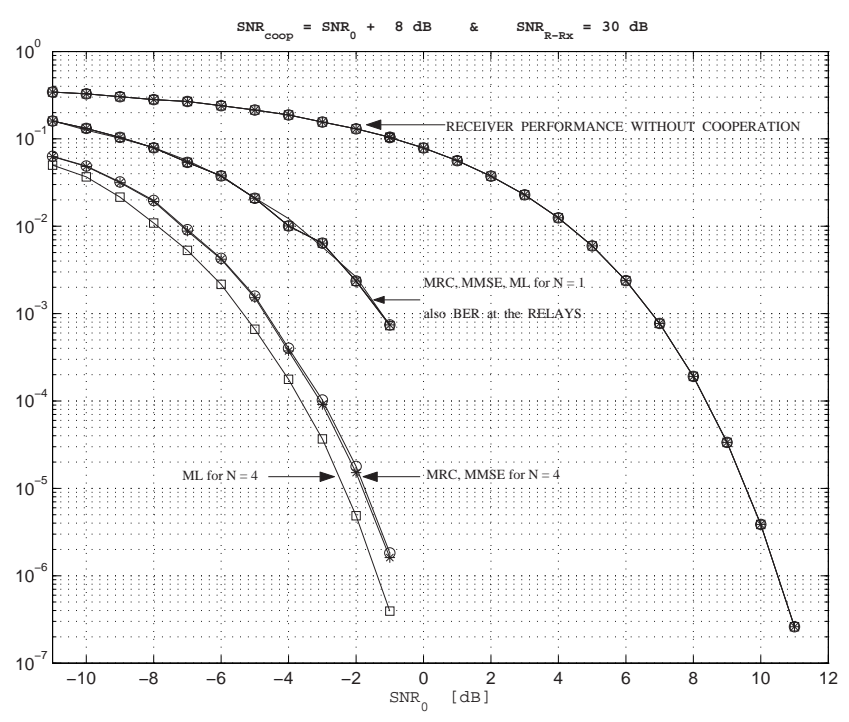

FIG. 5 - BER vs Rx SNR in the case of "good" relays

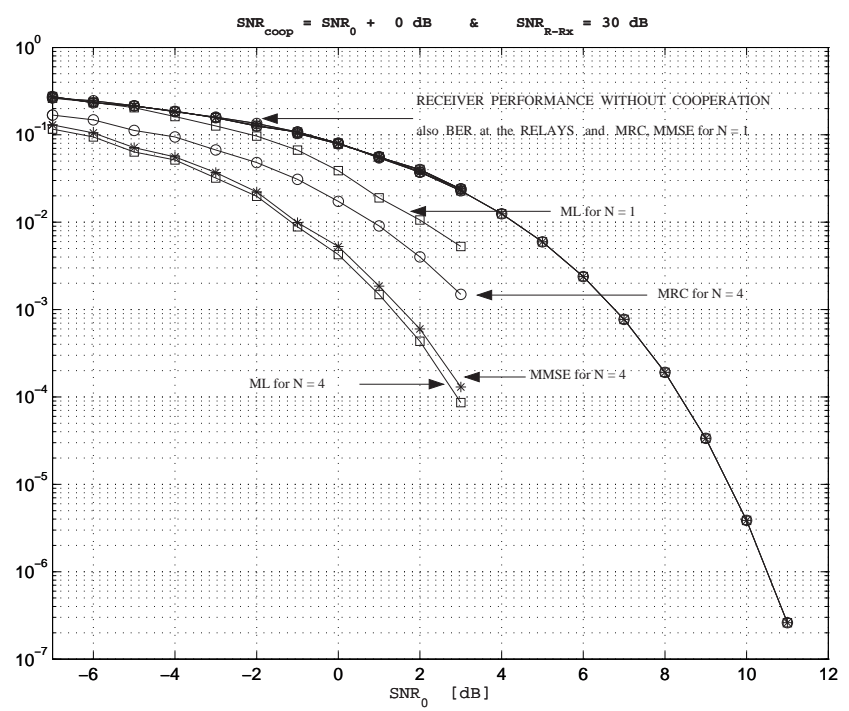

FIG. 6 - BER vs Rx SNR in the case of "medium" relays

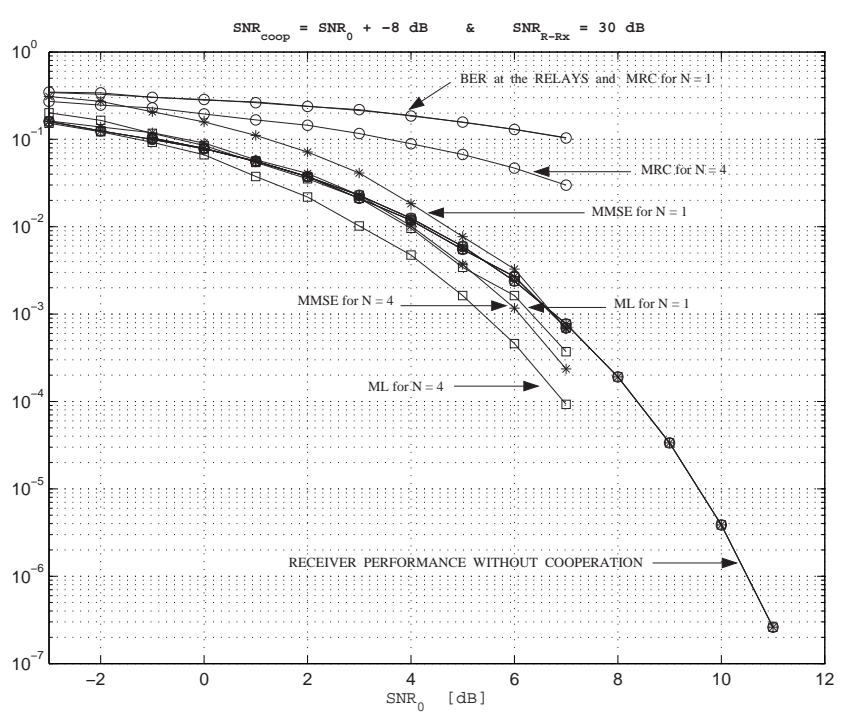

FIG. 7 - BER vs Rx SNR in the case of "bad" relays 